\section{Are cerebrospinal fluid protein levels and plasma neutrophil/lymphocyte ratio associated with prognosis of Guillain Barré syndrome?}

\author{
Sevki Sahin, Nilgun Cinar, \\ Sibel Karsidag \\ Department of Neurology, Maltepe \\ University, Istanbul, Turkey
}

\begin{abstract}
Guillain Barré syndrome (GBS) is a post-infectious acute autoimmune polyradiculopathy. Cerebrospinal fluid (CSF) total protein level and plasma neutrophil/lymphocyte ratio (NLR) are related with autoimmune response. We aimed to reach a prognostic indicator for GBS by using electrophysiological findings, protein level of CSF, and plasma NLR based on Medical Research Council (MRC) sum score data. Cases who met diagnostic criteria of GBS and followed at least six months were enrolled in the study. Nerve conduction study (NCS) and lumbar puncture were performed one week after symptom onset. Routine CSF findings and complete blood count were recorded. Plasma NLR was calculated as the ratio of neutrophil cell count to lymphocyte cell count. All patients
\end{abstract} received intravenous immunoglobulin. MRC sum scores were calculated on administration time (1st) and six months later (2nd) for evaluation of recovery. Mean values of baseline CSF protein level, NCS parameters and NLR were compared with mean scores of MRC1st and MRC ${ }^{2 n d}$. Increased CSF protein levels showed negative correlation with MRC2nd scores but no correlation with NCS. Increased NLR levels were positively correlated with age, MRC2nd scores and NCS. Facial diplegia was observed in $42 \%$ of patients. A positive correlation was found between high level of NLR and MRC1st, and there was no relationship with $\mathrm{MRC}^{2 n d}$. Regression analyses showed that only CSF protein level was an independent factor on both $\mathrm{MRC}^{1 \mathrm{st}}$ and $\mathrm{MRC}^{2 \mathrm{nd}}$. A positive association was found between baseline data included young age high plasma NLR, low level of CSF protein and good prognosis in our study. Also a positive correlation was found between high level of NLR and baseline disability in GBS cases with facial diplegia. Calculation of NLR is an easy and inexpensive method. On the other hand it may be influenced by age and immunotherapy. Our results showed that CSF protein level is still a liable parameter for prognosis. NLR could be a candidate prognostic marker of GBS cases. Further investigations including more cases are needed.

\section{Introduction}

Guillain Barré syndrome (GBS) is an acute disorder of the spinal nerve roots and peripheral nerves that is originated from immune mediated pathogenesis. ${ }^{1}$ Despite the new radiological and histopathological diagnostic techniques, nerve conduction studies (NCS) still provide best diagnostic clues in patients with GBS in clinical practice. $^{2}$

Total protein level of CSF suggests that the increased deposition of antibodies and complements and products of active myelin breakdown in inflammatory diseases of nervous system. ${ }^{3}$ In addition, elevated proteins have been shown as surrogate markers in the CSF for injury that correlate with disability and progression of demyelinating diseases. 4

Elevated neutrophil/lymphocyte ratio (NLR) may observe in chronic inflammatory diseases without significant leukocytosis in complete blood count. 5 Also the results of some previous studies about increased level of NLR indicate poor prognosis and high morbidity ratio in cardiovascular diseases and cancers. ${ }^{6}$ Although, significant correlation was found between elevated NLR and worse prognosis in Bell's palsy as a sample of inflammatory neuropathy in a study, ${ }^{7}$ there is no study that investigated the association between NLR and GBS in current literature yet. To investigate the prognostic markers of diseases has gained importance in recent years. Determination of prognosis in patients with GBS may provide preliminary information about treatment options, length of hospitalization and possible intensive care needs. ${ }^{8}$ The aim of this study is comparing CSF protein level, NLR level as inflammatory indicators and NCS with functional recovery scale (MRC sum score) as damage indicators to prediction to prognosis of GBS.

\section{Materials and Methods}

\section{Patients' selection}

This is a retrospective study of GBS cases that admitted to our clinic between 2011 and 2015. The diagnosis of GBS was made upon the criteria of Asbury and Cornblath. ${ }^{9}$ Detailed neurologic examination, routine biochemical investigations including complete blood count, glucose,
Correspondence: Sevki Sahin, Department of Neurology, Maltepe University, Faculty of Medicine, Maltepe, Istanbul, Turkey.

Tel: +90216440620 .

E-mail: drsahin@gmail.com

Conflict of interest: the authors declare no potential conflict of interest.

Contributions: SS, study desing; SS, NC, SK, acquisition of data and writing of the manuscript.

Key words: Polyradiculopathy; Marker; Inflammation; Nerve conduction study; Neutrophil/lymphocyte ratio.

Received for publication: 9 January 2017.

Revision received: 19 April 2017.

Accepted for publication: 22 April 2017.

This work is licensed under a Creative Commons Attribution NonCommercial 4.0 License (CC BY-NC 4.0).

(C) Copyright S. Sahin et al., 2017

Licensee PAGEPress, Italy

Neurology International 2017; 9:7032

doi:10.4081/ni.2017.7032

kidney and liver function tests, and cerebrospinal fluid analyses were done in all patients. Medical Research Council (MRC) sum score was performed to all the patients at on admission (MRC1st score) and 6months later (MRC2nd score). NCS findings, CSF protein and NLR data that recorded on first admission were compared withMRC 1 st and $\mathrm{MRC}^{2 \mathrm{nd}}$ score in order to show which factors affect clinical outcome. This study was approved by the institutional ethics committee.

\section{Medical research council sum score}

The degree of weakness was graded by using MRC score (ranging from 0 to 5) of six muscle pairs on both sides: upper arm abductors, elbow flexors, wrist extensors, hip flexors, knee extensors, and foot dorsiflexors. The MRC sum score ranges are from 0 (tetraplegia) to 60 (no paralysis). ${ }^{10}$

\section{Nerve conduction studies}

NCSs were performed one week after symptom onset in GBS cases by using Nihon Cohden Neuropack 2 system (NihonCohden Corp, Tokyo, Japan). Motor studies (distal motor latency, motor conduction velocity, F-wave and compound muscle action potential) were performed on both sides in the median, ulnar, tibial and peroneal nerves. Sensory nerve action potentials (SNAP) were done to assess sural, median and ulnar sensory nerves. The elec- 
trophysiological diagnosis of GBS was based upon the criteria suggested by Albers and Kelly. ${ }^{11}$ The ratio of proximal to distal amplitude of common peroneal nerve was calculated for all patients after stimulation. Conduction block was defined as ratio of proximal to distal amplitude $70 \%$ in the absence of temporal dispersion. All electrophysiological studies were performed from a board certified neurologist (SK, SS, NC). All testing was done while maintaining the skin temperature at $36^{\circ} \mathrm{C}$. We used the reference values that previously described by Oh for the nerve conduction studies. 12

\section{Examination of cerebrospinal fluid}

Spinal tap was performed in the supine position from lumbar levels using a standard 22 spinal needle, after one week from disease onset. CSF protein level of 45 $\mathrm{mg} / \mathrm{dL}$ was used as upper cutoff value of our laboratory. Cases with abnormal parameters of CSF except protein level were excluded.

\section{Calculation of neutrophil/lympho- cyte ratio}

NLR was calculated as the ratio of neutrophil cell count to lymphocyte cell count as previously described. 5

\section{Statistical analysis}

The statistical analysis was performed using the Statistical Package for Social Sciences, version 16 for windows (SPSS, Chicago, IL, USA). Kolmogorov-Smirnov test was used to test the normality of continuous variables and the variables with a $p$ value of more than 0.1 were regarded as normal distribution. Continuous variables with normal distribution were presented as mean \pm one standard deviation and analyzed by Mann-Whitney U test. The correlation between two continues variables was analyzed using the Spearman's correlation. In addition, a linear regression model was used to analyze the relationship between MRC score and age, CSF protein level, NLR. Statistical significance was defined at $\mathrm{P}$ value of 0.05 .

\section{Results}

Total 24 GBS cases (mean age: $41 \pm 16$, range18-73 years) were enrolled the study. Mean CSF protein level was found as $60 \pm 31 \mathrm{mg} / \mathrm{dL}$ (reference range: 0-45 $\mathrm{mg} / \mathrm{dL})$.

All patients received intravenous immunoglobulin (IVIG) $0.4 \mathrm{~g} / \mathrm{kg}$ for 5 days from day 1 to day 6 in hospitalization. Plasma exchange (PE) (ultrafiltration of 50 $\mathrm{mL} / \mathrm{kg}$ every other day-four sessions) required in two patients $(8.3 \%)$ after IVIG treatment because of progression (indication of assisted ventilation). Protein level of CSF was found as over $45 \mathrm{mg} / \mathrm{dL}$ in $92 \%$ of cases. A negative correlation between CSF protein level and MRC2nd score was found. NLR is negatively correlated with age and NCS parameters of median, ulnar and tibial motor nerves distal latencies and $\mathrm{F}$ latencies and it is positively correlated with MRC ${ }^{2 n d}$ score. The demographical, electrophysiological and biochemical data and correlation analyses of study group are given in Table 1.

Facial diplegia was observed in 10 patients $(42 \%)$. When compared facial diplegia with and without GBS cases, mean NLR level was higher and MRClst score (disability on admission) was lower in diplegia plus group (Table 2). Potential prognostic predictor for GBS cases were analyzed by regression analysis. This analysis showed that CSF protein level was found only significant predictor of prognosis when the scores of MRC1st andMRC2nd taken as a constant (Table 3 ).

\section{Discussion}

Guillain-Barré syndrome (GBS) is an acute inflammatory polyradiculoneuropathy that have distinct subtypes like acute motor axonal neuropathy (AMAN), acute inflammatory demyelinating polyneuropathy (AIDP) or acute motor and sensory axonal neuropathy (AMSAN). AIDP is the most common form of GBS and it is also called classical form. ${ }^{13}$ Our GBS group was established from electrophysiologically verified classic cases in order to reach homogeneous results.

GBS usually presents with bilateral symmetrical ascending flaccid paralysis but other unusual presentations such as cranial nerve palsy have been reported and among these, facial palsy is the most common (24$60 \%$ ). Bilateral simultaneous facial palsy is increasingly recognized as an atypical variant of GBS in adults. ${ }^{13,14}$ Facial diplegia ratio has been found as $42 \%$ in our study. When compared facial diplegia with and without GBS cases, NLR levels was higher and MRC1st score (disability on admission) was lower in diplegia plus group. Similar relation was not found with protein level of CSF and MRC1st score. It suggests that high NLR is related with initial disability but no related with prognosis. Ozler et al. showed a positive correlation between NLR values and grade of facial paralysis In Bell's palsy group. ${ }^{7}$ Our finding is similar with this study for our facial diplegia plus group.

Clinical manifestations of GBS cases vary depending on the severity of the infiltrative process in inflammatory neuropathies. ${ }^{15}$ The main pathological mechanism is macrophage infiltration and damage of the myelin sheath segment. Proximal nerve roots and intramuscular nerve sections are damaged more where the bloodnerve barrier is weaker. ${ }^{16} \mathrm{CSF}$ findings of GBS include that elevated protein, normal/slightly high lymphocytes $(<50$ cells $/ \mathrm{mm}^{3}$ ) cerebrospinal fluid analysis. This finding called albumino-cytologic dissociation (ACD) may be regarded as the first CSF bio-marker in GBS. It is presented in over 90 percent of patients. CSF protein level could be normal within the first week after disease onset, the elevated levels may be observed after 2-3 weeks. In a clinical trial, an increased total CSF protein levels have been determined in $50 \%$ of patients made of diagnostic lumbar puncture in the first week, $80 \%$ made of in the second week. ${ }^{17}$ We made CSF analyses into the first week of admission. Mean CSF protein level was found as $110 \pm 54 \mathrm{mg} / \mathrm{dL}$ and ACD was seen in $92 \%$ of cases in our study.

Total protein level of CSF suggests that the increased deposition of antibodies, complements and products of active myelin break down in inflammatory diseases of nervous system. ${ }^{3}$ Ritter et al. were evaluated IgG antibodies against the peripheral nerve tissue before and after IVIG treatment in their study. They found that the destructive antibody response was closely related with prognosis. ${ }^{18} \mathrm{We}$ found a negative effect of increased protein level of CSF in prognosis of GBS cases.

Various candidate biomarkers of CSF such as myelin basic protein, neuro-filaments, tau, anti-ganglioside antibodies, neuron specific enolase, hypocretin-1, 14-33 proteins, immunologic markers like different interleukins, tumor necrosis factor and complement system components for immune mediated polyneuropathies have been searched in previous studies. Some of these markers were suggested to have clinical relevance on prognosis of disease. However, studies on most CSF proteins are less, the numbers of patients are small. ${ }^{19} \mathrm{We}$ aimed to find out an easy and inexpensive prognostic marker for inflammatory polyneuropathies using the data obtained from routine clinical evaluation. Overall, we found a relationship between increased CSF protein levels with poor prognosis but plasma high NLR with good prognosis in GBS patients.

In a study of Ikincioglu et al., NLR and platelet-to-lymphocyte ratio (PLR) values were found to be significantly high in sudden sensorineural hearing loss (SSNHL) patients. In addition, their results suggested 
that baseline higher NLR values responded to the treatment better. ${ }^{20}$ These findings are similar to our results.

A poor prognosis in GBS has been correlated some parameters such as older age, ventilator necessity, preceding diarrhea or electrophysiological findings of axonal damage. ${ }^{19}$ We searched the role of CSF protein level and neutrophil/lymphocyte ratio as prognostic markers of outcome and electrophysiological findings. In our study, NLR value that considered an inflammation marker in recent years was negatively correlated with age in our study group. Some authors reported that the positive relationship between NLR and increased age.5,21 Mean age of our group was 41 years. If our study included children or elders, our results would be completely different.

Elevated NLR may observe in chronic inflammatory diseases. ${ }^{19}$ A high NLR independently predicts poor survival in patients with unresectable hepatocellular carcinoma undergoing transarterial chemoembolization treatment, and an increased NLR indicates a better outcome than a decreased NLR for patients after transarterial chemoembolization.22 We gave immunotherapy to all patients. This may explain the relationship that we found between high levels of NLR and good prognosis. If our study included a patient group who was not given immunotherapy, our results would be different. But this is not ethical for GBS patients.

\section{Conclusions}

Our results showed that the high levels of baseline NLR indicate good prognosis for all cases in correlation analyses. In facial diplegia positive group, despite a positive correlation was found between high level of NLR and baseline disability on admission, there was no relationship with prognosis. Regression analysis showed that the protein level CSF was an independent prognostic factor. These contradictory results might be related to the following limitations. The first one, CSF analysis and NCS examination is only made into the first week in our study. However, second assessment of functional recovery was made by MRC scale only after six months. The second limitation is the relatively small sample size of our study group. Third one is immunotherapy, which was given all patients and it could influenced the natural changing of NLR

Table 1. Correlation analyses of data.

\begin{tabular}{|c|c|c|c|}
\hline \multirow{2}{*}{ Parameter of GBS cases } & \multirow[t]{2}{*}{ Mean \pm SD $(\min -\max )(\mathrm{n}=24)$} & \multicolumn{2}{|c|}{ Correlation analyses } \\
\hline & & CSF protein & NLR \\
\hline Age (years) & $41 \pm 16(18-73)$ & 0.1 & $-0.007^{*}$ \\
\hline $\begin{array}{l}\text { Median motor nerve } \\
\text { Distal latency (ms) } \\
\text { Amplitude (mV) } \\
\text { Conduction velocity (m/s) } \\
\text { Min F latency (ms) } \\
\end{array}$ & $\begin{array}{c}4.9 \pm 2(2.5-10.5) \\
4.5 \pm 3(0.05-14.2) \\
45 \pm 15(20-68) \\
36.2 \pm 8(29-53)\end{array}$ & $\begin{array}{l}0.3 \\
0.8 \\
0.5 \\
0.2\end{array}$ & $\begin{array}{c}0.6 \\
0.01^{*} \\
0.9 \\
0.2\end{array}$ \\
\hline $\begin{array}{l}\text { Ulnar motor nerve } \\
\text { Distal latency (ms) } \\
\text { Amplitude (mV) } \\
\text { Conduction velocity (m/s) } \\
\text { Min F latency (ms) }\end{array}$ & $\begin{array}{l}3.2 \pm 1(2.1-6.4) \\
4.6 \pm 1.9(2-7.3) \\
48 \pm 10(22-60) \\
34.1 \pm 9(28-49)\end{array}$ & $\begin{array}{l}0.1 \\
0.4 \\
0.2 \\
0.7\end{array}$ & $\begin{array}{c}-0.05^{*} \\
0.1 \\
0.7 \\
-0.04^{*}\end{array}$ \\
\hline $\begin{array}{l}\text { Peroneal motor nerve } \\
\text { Distal latency (ms) } \\
\text { Amplitude (mV) } \\
\text { Conduction velocity (m/s) } \\
\text { Min F latency (ms) } \\
\end{array}$ & $\begin{array}{c}5.8 \pm 2(3.6-12) \\
1.5 \pm 1.2(0.1-4.7) \\
38.3 \pm 10(19-57) \\
60 \pm 16(43-88)\end{array}$ & $\begin{array}{l}0.3 \\
0.3 \\
0.5 \\
0.7\end{array}$ & $\begin{array}{c}0.5 \\
0.4 \\
0.08 \\
0.1\end{array}$ \\
\hline $\begin{array}{l}\text { Tibial motor nerve } \\
\text { Distal latency (ms) } \\
\text { Amplitude (mV) } \\
\text { Conduction velocity (m/s) } \\
\text { Min F latency (ms) }\end{array}$ & $\begin{array}{c}7 \pm 2.6(4-12.4) \\
2.6 \pm 2.2(0.4-8.4) \\
38.5 \pm 10(22-60) \\
58 \pm 15(37-101)\end{array}$ & $\begin{array}{l}0.3 \\
0.9 \\
0.5 \\
0.9\end{array}$ & $\begin{array}{c}0.4 \\
0.3 \\
0.002^{*} \\
-0.04^{*}\end{array}$ \\
\hline $\begin{array}{l}\text { Median sensory nerve } \\
\text { Onset latency }(\mathrm{ms}) \\
\text { Amplitude }(\mu \mathrm{V}) \\
\text { Conduction velocity }(\mathrm{m} / \mathrm{s})\end{array}$ & $\begin{array}{l}2.5 \pm 0.4(1.8-3.4) \\
22.6 \pm 20(4.2-68) \\
\quad 46 \pm 7(37-59) \\
\end{array}$ & $\begin{array}{l}0.8 \\
0.7 \\
0.4\end{array}$ & $\begin{array}{l}0.4 \\
0.9 \\
0.5\end{array}$ \\
\hline $\begin{array}{l}\text { Ulnar sensory nerve } \\
\text { Onset latency (ms) } \\
\text { Amplitude }(\mu \mathrm{V}) \\
\text { Conduction velocity }(\mathrm{m} / \mathrm{s})\end{array}$ & $\begin{array}{c}2 \pm 0.3(1.4-2.4) \\
18.7 \pm 13(2.4-51) \\
49 \pm 6(39-60.8)\end{array}$ & $\begin{array}{c}0.08 \\
0.6 \\
0.2\end{array}$ & $\begin{array}{l}0.5 \\
0.7 \\
0.8\end{array}$ \\
\hline $\begin{array}{l}\text { Sural sensory nerve } \\
\text { Onset latency (ms) } \\
\text { Amplitude }(\mu \mathrm{V}) \\
\text { Conduction velocity (m/s) } \\
\text { CSF protein level (mg/dL) } \\
\text { Plasma NLR } \\
\text { MRC1st score (min max) } \\
\text { MRC2st score (min max) }\end{array}$ & $\begin{array}{c}2.8 \pm 0.9(1.6-4.6) \\
9.6 \pm 6(3.2-26.9) \\
41 \pm 7(28-54.9) \\
110 \pm 54(49-250) \\
2.6 \pm 1.1(1.2-4.7) \\
47 \pm 6(38-56) \\
56 \pm 3(50-60)\end{array}$ & $\begin{array}{c}0.06 \\
0.3 \\
0.7 \\
- \\
-0.06 \\
0.1 \\
-0.01 *\end{array}$ & $\begin{array}{c}0.4 \\
0.2 \\
0.07 \\
-0.06 \\
- \\
0.6 \\
0.007^{*}\end{array}$ \\
\hline
\end{tabular}

GSB, Guillain Barré syndrome; SD, standard deviation; CSF, cerebrospinal fluid; NLR, neutrophil/lymphocyte ratio; MRC, Medical Research Council. *P<0.05. 
Table 2. The comparative analyses of facial diplegia (+) and (-) group.

\begin{tabular}{lccc} 
& Facial diplegia $(+) /(-)$ & Mean \pm SD & P \\
Age (years) & $(+)$ & $39 \pm 15$ & 0.6 \\
& $(-)$ & $43 \pm 17$ & \\
CSF protein level (mg/dL) & $(+)$ & $134 \pm 66$ & 0.3 \\
& $(-)$ & $93 \pm 37$ & \\
\hline Plasma NLR & $(+)$ & $3.1 \pm 1.2$ & $0.03^{*}$ \\
MRC1st score & $(-)$ & $2 \pm 0.5$ & \\
& $(+)$ & $44 \pm 5$ & $0.01^{*}$ \\
\hline MRC2nd score & $(-)$ & $50 \pm 6$ & \\
& $(+)$ & $57 \pm 4$ & 0.3 \\
\hline
\end{tabular}

SD, standard deviation; CSF, cerebrospinal fluid; NLR, neutrophil/ymphocyte ratio; MRC, Medical Research Council. ${ }^{*} \mathrm{P}<0.05$.

Table 3. Regression analyses to find prognostic predictor for Guillain Barré syndrome cases.

\begin{tabular}{lcccc} 
Model & \multicolumn{2}{c}{$\begin{array}{c}\text { Unstandardized } \\
\text { coefficients }\end{array}$} & $\begin{array}{c}\text { Standardized } \\
\text { coefficients }\end{array}$ & P \\
Constant: MRC1st score & 59,985 & SE & & \\
NLR & -2.007 & 6,370 & - & 0.000 \\
CSF protein level & -0.087 & 1.199 & -0.388 & 0.118 \\
Age & 0.075 & 0.020 & -0.890 & $0.001^{*}$ \\
Constant: MRC2nd score & 61,418 & 0.085 & 0.205 & 0.390 \\
NLR & 0.641 & 3,324 & - & 0.000 \\
CSF protein level & -0.031 & 0.625 & 0.205 & 0.324 \\
Age & -0.065 & 0.010 & -0.517 & 0.011 \\
\hline
\end{tabular}

SE, standard error; MRC, Medical Research Council; NLR, neutrophil/ymphocyte ratio; CSF, cerebrospinal fluid. ${ }^{*} \mathrm{P}<0.05$.

levels. On the other hand, our groups were consisted from homogenous classic form of GBS cases included facial diplegia positive group. This feature enabled us to achieve clear interpretable data. To the best of our knowledge, our study is the first prognostic study of GBS based on NCS, CSF protein level and plasma NLR. We showed that the parameters that can reproduce from routine analysis could be valuable about prognosis. Further studies including a larger patient population combined with other biomarkers are needed.

\section{References}

1. Steiner IG Rosenberg G, Wirguin I. Transient immunosuppression: a bridge between infection and the atypical autoimmunity of Guillain-Barré syndrome? Clin Exp Immunol 2010;162: $32-40$.

2. Chichkova RI, Katzin L. Emg and nerve conduction studies in clinical practice. Pract Neurol 2010;2010:32-38.

3. Goverman J. Autoimmune T cell responses in the central nervous system. Nat Rev Immunol 2009;9:393-407.

4. Kottil WR. Cerebrospinal fluid in mul- tiple sclerosis. Ann Indian Acad Neurol 2009; 12:246-53.

5. Seals DR, Kaplon RE, Gioscia-Ryan RA, LaRocca TJ. You're only as old as your arteries: translational strategies for preserving vascular endothelial function with aging. Physiology 2014;29:250-64.

6. Imtiaz F, Shafique K, Mirza SS, et al. Neutrophil lymphocyte ratio as a measure of systemic inflammation in prevalent chronic diseases in Asian population. Int Arch Med 2012:26;5:2.

7. Ozler GS, Gunak G. Neutrophil-lymphocyte ratio: a new predictive and prognostic factor in patients with Bell palsy. J Craniofac Surg 2014;25:944-5.

8. van Doorn PA, Ruts L, Jacobs BC. Clinical features, pathogenesis, and treatment of Guillain-Barré syndrome. Lancet Neurol 2008;7:939-950.

9. Asbury AK, Cornblath DR. Assessment of current diagnostic criteria for Guillain-Barre syndrome. Ann Neurol 1990;27(Suppl.1):21-4.

10. Kleyweg RP, van der Meche FGA, Schmitz PIM. Interobserver agreement in the assessment of muscle strength and functionalabilities in GuillainBarré syndrome. Muscle Nerve 1991;
14:1103-9.

11. Albers JW, Kelly JJ Jr. Acquired inflammatory demyelinating polyneuropathies: clinical and electrodiagnostic features. Muscle Nerve 1989;12:43551.

12. Oh SJ. Nerve conduction studies. In: Oh SJ (ed) Clinical electromyography, 2nd edn. Williams \&Wilkins, Baltimore, MD, USA, 1993, pp 270-2.

13. Atsumi M, Kitaguchi M, Nishikawa S, Susuki K. A variant of Guillain-Barré syndrome with prominent bilateral peripheral facial nerve palsy-facial diplegia and paresthesias. Rinsho Shinkeigaku 2004;44:549-52.

14. Narayanan RP, James N, Ramachandran K, Jaramillo MJ. Guillain-Barré syndrome presenting with bilateral facial nerve paralysis: a case report. Cases J 2008;8:379.

15. Hughes RA, Cornblath DR. GuillainBarré syndrome. Lancet 2005;5: 366:1653-66.

16. Willison HJ. Ganglioside complexes: new autoantibody targets in GuillainBarré syndromes. Nat Clin Pract Neurol 2005;1:2-3.

17. van den Berg B, Walgaard C, Drenthen $\mathrm{J}$, et al. Guillain-Barré syndrome: pathogenesis, diagnosis, treatmentandprognosis. Nat Rev Neurol 2014;10:46982.

18. Ritter C, Bobylev I, Lehmann HC. Chronic inflammatory demyelinating polyneuropathy (CIDP): Change of serum IgG dimer levels during treatment with intravenous immunoglobulins. J Neuroinflammation 2015;12:148.

19. Sung EJ, Kim DY, Chang MC, Ko EJ. Prediction of functional outcome in axonal Guillain-Barre syndrome. Ann Rehabil Med 2016;40:481-8.

20. Ikinciogulları A, Koseoglu S, Kilic M, et al. New inflammation parameters in sudden sensorineural hearing loss: neutrophil-to-lymphocyte ratio and platelet-to-lymphocyte ratio. Int $\mathrm{Adv}$ Otol 2014;10:197-200.

21. Li J, Chen Q, Luo X, Hong J, Pan K, Lin X, Liu X, Zhou L, Wang H, Xu Y, Li H, Duan C. Neutrophil-tolymphocyte ratio positively correlates to age in healthy population. J Clin Lab Anal 2015;29:437-43.

22. Huang ZL, Luo J, Chen MS, et al. Blood neutrophil-to-lymphocyte ratio predicts survival in patients with unresectable hepatocellular carcinoma undergoing transarterial chemoembolization. J Vasc Interv Radiol 2011;22: 702-9. 